\title{
A New Technique to Extract the Gate Bias Dependent S/D Series Resistance of Sub-100nm MOSFETs
}

\author{
Dominique Fleury ${ }^{1,2}$, Antoine $\operatorname{Cros}^{1}$, Grégory Bidal ${ }^{1,2}$, Hugues Brut ${ }^{1}$, Emmanuel Josse $^{1}$ and Gérard Ghibaudo ${ }^{2}$ \\ ${ }^{1}$ STMicroelectronics, 850 rue Jean Monnet 38926 Crolles cedex, France \\ 2 IMEP-LAHC, 3 rue Parvis Louis Néel BP 25738016 Grenoble cedex 1 France \\ Tel. : +33-4-3892-3314, Fax : +33-4-3892-2953, email : dominique.fleury@ st.com
}

\begin{abstract}
In this study, a new technique to extract the S/D series resistance $\left(\mathrm{R}_{\mathrm{sd}}\right)$ from the total resistance versus transconductance gain plot $\mathrm{R}_{\mathrm{tot}}(1 / \beta)$ is proposed. The technique only requires the measurement of $\left.\mathrm{I}_{\mathrm{d}}\left(\mathrm{V}_{\mathrm{gs}}\right)\right|_{\mathrm{Vgt}}$ and $\beta$, allowing fast and statistical analysis in an industrial context. Unlike the usual $\mathrm{R}_{\mathrm{tot}}(\mathrm{L})$-based techniques, it has the advantage of being insensitive to the channel length and mobility variations and finally enables to extract very accurate values for $R_{s d}\left(V_{g s}\right)$ and the effective mobility reduction factor $\mu_{\text {eff }}\left(V_{\mathrm{gt}}\right) / \mu_{\mathrm{eff}}(0)$.
\end{abstract}

\section{INTRODUCTION}

The S/D resistance $\left(R_{s d}\right)$ is a major concern for the MOSFET scaling as it plays a key role in device performance and power consumption [1]. Since the channel length is scaled down, the $\mathrm{R}_{\mathrm{sd}} / \mathrm{R}_{\text {tot }}$ ratio becomes higher and $R_{s d}$ requires improved accuracy in extraction techniques to be assessed within a reasonable error. As described on Fig.1, a transistor can be modeled in linear regime by a channel resistance $R_{c h}$ connected to the $S / D$ series resistance $R_{s d}=R_{s}+R_{d}$ through which the drain current $I_{d}$ flows $\left(R_{t o t}=R_{s d}+R_{c h}\right)$. Due to pockets implants, strain booster and neutral defects, the effective mobility $\left(\mu_{\text {eff }}\right)$ changes as a function of channel length ( $\mathrm{L}_{\mathrm{eff}}$ ) [2]-[4] (Fig.2). As a consequence, $\mathrm{R}_{\mathrm{ch}}$ is no more strictly proportional to the geometrical dimensions of the channel and all $R_{\text {tot }}(L)$-based techniques [5-7] fail when $\mu_{\text {eff }}(L)$ variations are not properly compensated for [8] (cf. Fig.3). To solve this issue, a new extraction technique based on the relationship between $\mathrm{R}_{\text {tot }}$ and the transconductance gain $\beta$ of the transistor in linear regime is proposed. The technique is insensitive to the $\mu_{\text {eff }}(\mathrm{L}), \mathrm{L}_{\mathrm{eff}}(\mathrm{L})$ variations (which generally make the other techniques inaccurate) and provides a straightforward way to extract $R_{\text {sd }}$ statistically.

\section{THE $\mathrm{R}_{\text {Tот }}(1 / \beta)$ TECHNIQUE}

The $\mathrm{R}_{\mathrm{tot}}(1 / \beta)$ technique relies on the BSIM3v3 model (1) which reproduces the drain current behavior in linear regime. In $(1), \mathrm{V}_{\mathrm{gt}}=\left(\mathrm{V}_{\mathrm{gs}}\right.$ $\left.-V_{t h}\right)$ is the gate overdrive, $\beta=\mu_{\text {eff }}(0) . C_{o x} \cdot W_{\text {eff }} / L_{\text {eff }}$ is the transconductance gain (where $\mu_{\text {eff }}(0)$ is the effective mobility extrapolated to $\mathrm{V}_{\mathrm{gt}}=$ $0 \mathrm{~V})$ and $\left(\Theta_{1}, \Theta_{2}\right)$ are the first and second order mobility attenuation factors, respectively.

$$
I_{d}=\mu_{\text {eff }} C_{o x} \frac{W}{L} V_{g t}\left(V_{d s}-R_{s d} I_{d}\right)=\frac{\beta V_{d s} V_{g t}}{1+\Theta_{1} V_{g t}+\Theta_{2} V_{g t}^{2}}
$$

The channel resistance is defined as $\mathrm{R}_{\mathrm{ch}}=\mathrm{V}_{\mathrm{d}, 0} / \mathrm{I}_{\mathrm{d}, 0}$ (where the " 0 " subscript refers to the intrinsic value of the parameter, for $R_{s d}=0$ $\Omega . \mu \mathrm{m})$. From (1), $\mathrm{R}_{\mathrm{tot}}$ can be expressed as (2).

$$
\mathrm{R}_{\mathrm{tot}}=\frac{1}{\beta} \cdot\left(\frac{1+\Theta_{1,0} \mathrm{~V}_{\mathrm{gt}}+\Theta_{2,0} \mathrm{~V}_{\mathrm{gt}}{ }^{2}}{\mathrm{~V}_{\mathrm{gt}}}\right)+\mathrm{R}_{\mathrm{sd}}\left(\mathrm{V}_{\mathrm{gs}}\right)
$$

When $\mathrm{V}_{\mathrm{gt}}$ is fixed once for a full set of devices with several channel lengths, the $R_{\text {tot }}=f(1 / \beta)$ plot shows a linear behavior which returns the mobility reduction from the slope (3) and the $R_{\mathrm{sd}} \mid V_{\mathrm{gt}}$ from the $\mathrm{y}$-axis intercept (2). By repeating the same extraction for several gate overdrives, $\mathrm{R}_{\mathrm{sd}}\left(\mathrm{V}_{\mathrm{gs}}\right)$ and $\mu_{\mathrm{eff}}\left(\mathrm{V}_{\mathrm{gt}}\right) / \mu_{\mathrm{eff}}(0)$ can be extracted

$$
\left.\mathrm{V}_{\mathrm{gt}} \cdot \frac{\partial \mathrm{R}_{\mathrm{tot}}}{\partial(1 / \beta)}\right|_{\mathrm{V}_{\mathrm{gt}}}=1+\Theta_{1,0} \mathrm{~V}_{\mathrm{gt}}+\Theta_{2,0} \cdot \mathrm{V}_{\mathrm{gt}}{ }^{2}=\frac{\mu_{\mathrm{eff}}(0)}{\mu_{\mathrm{eff}}\left(\mathrm{V}_{\mathrm{gt}}\right)}
$$

\section{RESULTS}

The following results were obtained by measurements on our $45 \mathrm{~nm}$ node technology platform on the low stand-by power devices, featuring
$1.7 \mathrm{~nm}$-EOT SiON gate dielectric with polysilicon gate and tensile contact etch stop layer for nMOS mobility optimization [9] (Fig.1). Extraction also been performed on FDSOI devices featuring metal gate (WN) with $2.5 \mathrm{~nm}$ EOT $\mathrm{HfSi}_{\mathrm{x}} \mathrm{O}_{\mathrm{y}} \mathrm{N}_{\mathrm{z}}$ dielectric, $12 \mathrm{~nm}$ thinned $\mathrm{Si}$ film and elevated $S / D$ [10]. Statistical $\mathrm{I}_{\mathrm{d}}\left(\mathrm{V}_{\mathrm{gs}}\right)$ measurements (72 dices) have been performed for lengths ranging from $35 \mathrm{~nm}$ to $240 \mathrm{~nm}$ and $\mathrm{W}=1 \mu \mathrm{m}$. Strong pockets implants have been used in the process to increase the channel doping and limit the short channel effect in the smallest devices. $V_{\text {th }}$ and $\beta$ can be extracted from the McLarty's function [11] (4) or from the $\xi$-function [12] which have both the advantage of being insensitive to $\left(\Theta_{1}, \Theta_{2}\right)$ when $R_{\mathrm{sd}}$ has a linear variation with $\mathrm{V}_{\mathrm{gs}}$.

$$
\left(\frac{\partial^{2} \mathrm{R}_{\mathrm{tot}}}{\partial \mathrm{V}_{\mathrm{gs}}^{2}}\right)^{-1 / 3}=\left(\frac{\beta}{2}\right)^{1 / 3} \mathrm{~V}_{\mathrm{gt}}
$$

Note that, as displayed in the inset of Fig.4, $\mathrm{V}_{\text {th }}$ deduced from McLarty's functions and $\xi$-function corresponds to the charge threshold voltage at strong inversion i.e. where $\mathrm{Q}_{\text {inv }}=\mathrm{C}_{\mathrm{ox}} \cdot \mathrm{V}_{\mathrm{gt}} \cdot \mathrm{V}_{\mathrm{th}}\left(\mathrm{L}_{\mathrm{eff}}\right)$ and $\beta\left(L_{\text {eff }}\right)$ behavior are displayed on Fig.4 and Fig.5, where $L_{\text {eff }}$ has been extracted from C-V measurements [13]. $\mathrm{R}_{\mathrm{tot}}$ has been measured for each device at several gate overdrive ranging from 0.1 to $1.1 \mathrm{~V}$ (the nominal voltage for this technology is $\mathrm{V}_{\mathrm{gs}}=1.1 \mathrm{~V}$, i.e. $\mathrm{V}_{\mathrm{gt}} \approx 0.4 \mathrm{~V}$ ). $\mathrm{R}_{\mathrm{sd}}\left(\mathrm{V}_{\mathrm{gt}}\right)$ has been extracted from the $\mathrm{R}_{\mathrm{tot}}=\mathrm{f}(1 / \beta)$ plot, as described previously (2). The linear regression is displayed on Fig.5, where data has been filtered with a recursive normal filter within a $\pm 3 \sigma$-tolerance $(99 \%$ confidence). The points show a very good alignment which results in a very small error on the final result: $R^{2}>0.99, R_{s d}=(110 \pm 3) \Omega . \mu m$. Fig.7 shows $\mathrm{R}_{\mathrm{sd}}\left(\mathrm{V}_{\mathrm{gs}}\right)$, where $\mathrm{V}_{\mathrm{gs}}$ has been approximated to $\mathrm{V}_{\mathrm{gs}} \approx \mathrm{V}_{\mathrm{gt}}+\left\langle\mathrm{V}_{\mathrm{th}}(\mathrm{L})\right\rangle,\left\langle\mathrm{V}_{\mathrm{th}}(\mathrm{L})\right\rangle$ being the average $\mathrm{V}_{\text {th }}$ for the set of devices: $\mathrm{V}_{\mathrm{gs}} \approx \mathrm{V}_{\mathrm{gt}}+0.69 \pm 0.05 \mathrm{~V}$ (cf. Fig.4). The behavior of $\mathrm{R}_{\mathrm{sd}}\left(\mathrm{V}_{\mathrm{g}}\right)$ is consistent with previous studies [14]. Results extracted for small gate overdrive $\left(\mathrm{V}_{\mathrm{gt}} \leq 0.2 \mathrm{~V}\right)$ show a slight deviation, which might be due to the limited accuracy in the $\mathrm{V}_{\mathrm{th}}$-extraction technique and/or non validity of strong inversion approximation close to $\mathrm{V}_{\mathrm{th}}$. Intrinsic mobility reduction factors have been extracted from (3) to be compared with the $\Theta(\beta)$ technique [15],[16]. As shown on Fig.8 and Fig.9, both techniques provide very close $\Theta_{1,0}$ values but $R_{\text {sd }}$ extracted from $\Theta(\beta)$ shows a larger dispersion mainly induced by uncertainties on the $\Theta_{1}$ parameter extraction. Finally, error resulting from the $\left\langle\mathrm{V}_{\mathrm{th}}(\mathrm{L})\right\rangle$ approximation has also been quantified (Fig10) and $\mathrm{R}_{\mathrm{sd}}$ has been estimated for the two extraction techniques. Results for bulk and FDSOI MOSFETs are summarized in Tab.1. As expected, FDSOI devices benefit from a lowered $R_{s d}$ thanks to the elevated epitaxial S/D and an improved accuracy is confirmed for the $R_{t o t}(1 / \beta)$ technique compared to the $\Theta(\beta)$ one.

\section{CONCLUSION AND PERSPECTIVES}

This study demonstrates the ability of a new $R_{\text {tot }}(1 / \beta)$ technique to provide $\mathrm{R}_{\mathrm{sd}}\left(\mathrm{V}_{\mathrm{g}}\right)$ and $\mu_{\mathrm{eff}}\left(\mathrm{V}_{\mathrm{gt}}\right) / \mu_{\text {eff }}(0)$ values with an improved accuracy thanks to statistical results. Unlike the $\mathrm{R}_{\text {tot }}(\mathrm{L})$-based technique, the use of $1 / \beta$ for the $x$-axis allows to correct any $\mu_{\text {eff }}$ or $L_{\text {eff }}$ variations. The technique only requires to measure $\left.\mathrm{I}_{\mathrm{d}}\left(\mathrm{V}_{\mathrm{gs}}\right)\right|_{\mathrm{Vgt}}$ and $\beta$ on several channel lengths. The results match with the $\Theta(\beta)$ technique which suffers from a larger dispersion and requires full $\mathrm{I}_{\mathrm{d}}\left(\mathrm{V}_{\mathrm{gs}}\right)$-curves measurements to extract $R_{s d}$. this technique is fully compatible with fast measurement techniques, offering new perspectives towards $\mathrm{R}_{\mathrm{sd}}$ monitoring and large scale analysis in industrial environment.

\section{ACKNOWLEDGMENTS}

The authors would like to thank the Advanced Modules and Process Integration teams for providing the devices used in this work. 


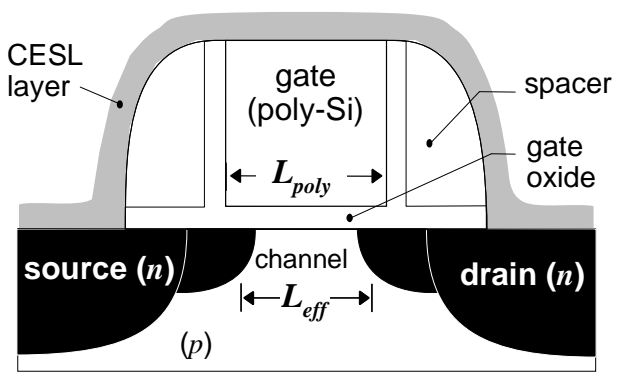

$\mathrm{V}_{\mathrm{s}}$

Fig.1 - Typical bulk nMOSFET with tensile contact etch stop layer (CESL).

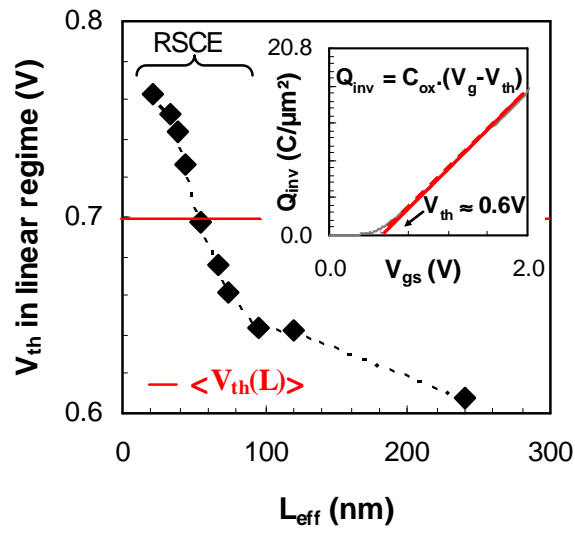

Fig.4 $-V_{\text {th }}\left(L_{\text {eff }}\right)$ plot for nMOSFETs in linear regime, in inset: definition of $\mathrm{V}_{\mathrm{th}}$.

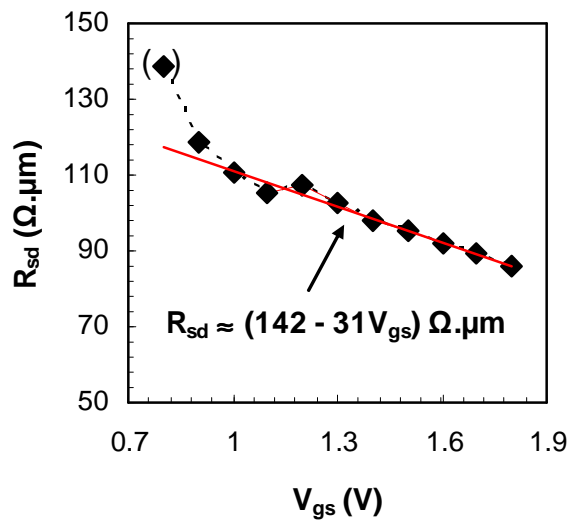

Fig.7 $-\mathrm{R}_{\mathrm{sd}}\left(\mathrm{V}_{\mathrm{g}}\right)$ behaviour extracted from the $\mathrm{R}_{\mathrm{tot}}(1 / \beta)$ technique.

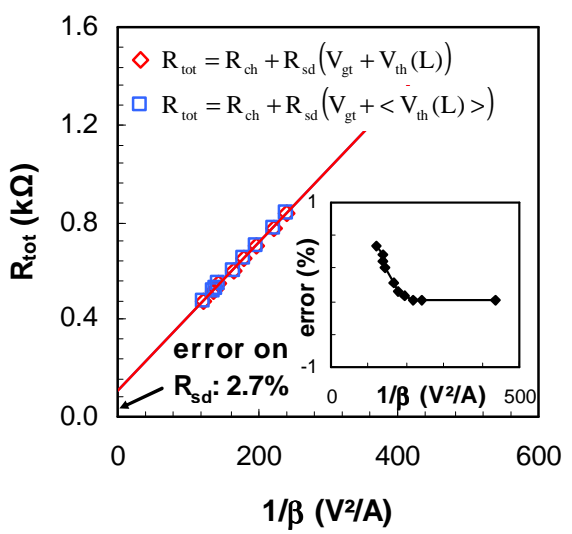

Fig.10 - Comparison between exact model $(\diamond)$ and approximation $(\square)$ using $\left\langle\mathrm{V}_{\mathrm{th}}(\mathrm{L})\right\rangle$

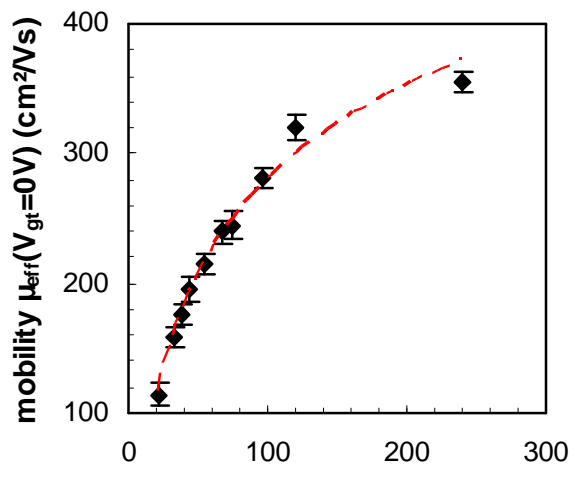

effective channel length $L_{\text {eff }}(\mathrm{nm})$

Fig.2 - decrease of the low field mobility for short channel length nMOSFETs

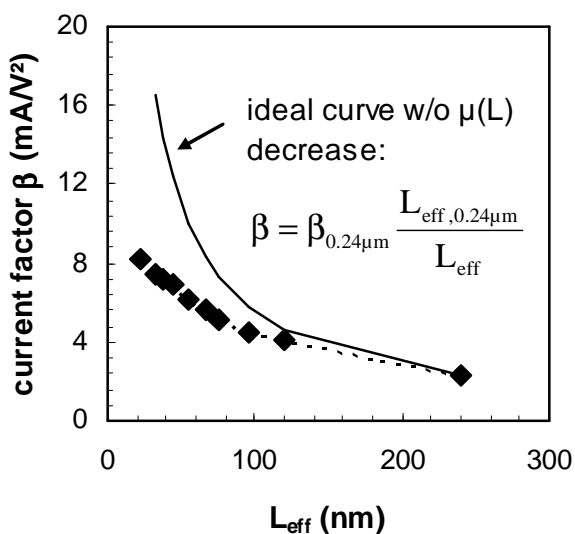

Fig.5 - $\beta\left(\mathrm{L}_{\mathrm{eff}}\right)$ measurements. Continuous line: Fig.6 $-\mathrm{R}_{\mathrm{tot}}(1 / \beta)$ plot for nMOSFETs. $\mathrm{R}_{\mathrm{sd}}$ is ideal behaviour w/o mobility reduction

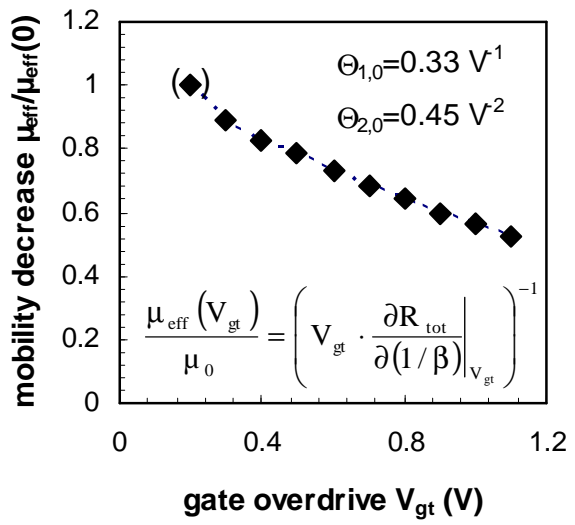

Fig.8 - Mobility decrease (as a function of $\mathrm{V}_{\mathrm{gs}}$ ) from the $\mathrm{R}_{\mathrm{tot}}(1 / \beta)$ technique.

\begin{tabular}{|r|c|c|}
\hline $\mathbf{R}_{\text {sd }}(\boldsymbol{\Omega} . \boldsymbol{\mu m})$ & $\mathbf{R}_{\text {tot }}(\mathbf{1} / \boldsymbol{\beta})$ & $\boldsymbol{\Theta}(\boldsymbol{\beta})$ \\
\hline nMOS bulk & $110 \pm 3$ & $119 \pm 10$ \\
\hline pMOS bulk & $170 \pm 5$ & $155 \pm 15$ \\
\hline nMOS FDSOI & $97 \pm 5$ & $126 \pm 34$ \\
\hline pMOS FDSOI & $156 \pm 5$ & $208 \pm 50$ \\
\hline
\end{tabular}

Tab.1 $-\mathrm{R}_{\mathrm{sd}} \mid \mathrm{V}_{\mathrm{gs}}=1.1 \mathrm{~V}$ values extracted for bulk and FDSOI MOSFETs and compared to results obtained from the $\Theta(\beta)$ technique. As expected, the $\mathrm{R}_{\mathrm{tot}}(1 / \beta)$ method gives more accurate results which remain in line with $\Theta(\beta)$.

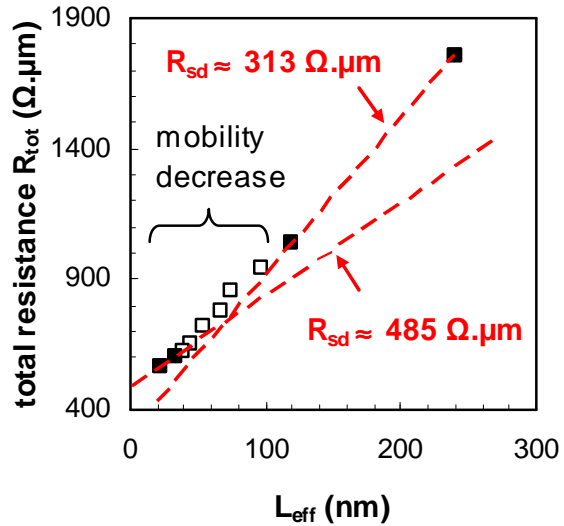

Fig.3 - uncertainty on the $\mathrm{R}_{\text {tot }}(\mathrm{L})$ technique due to $\mu(\mathrm{L})$ degradation on short channels

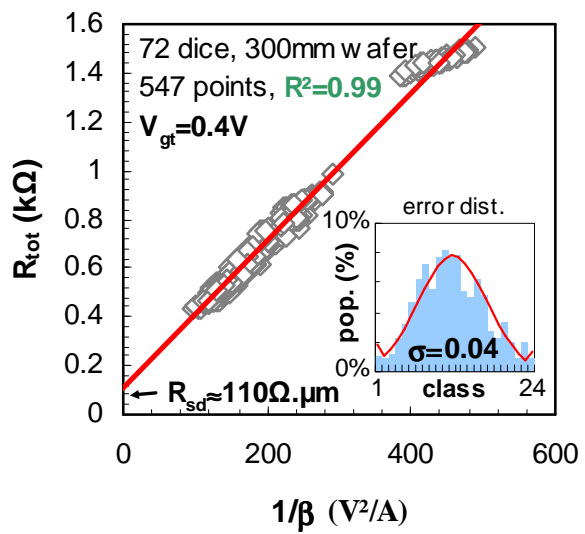
extracted from the intercept with the $y$-axis.

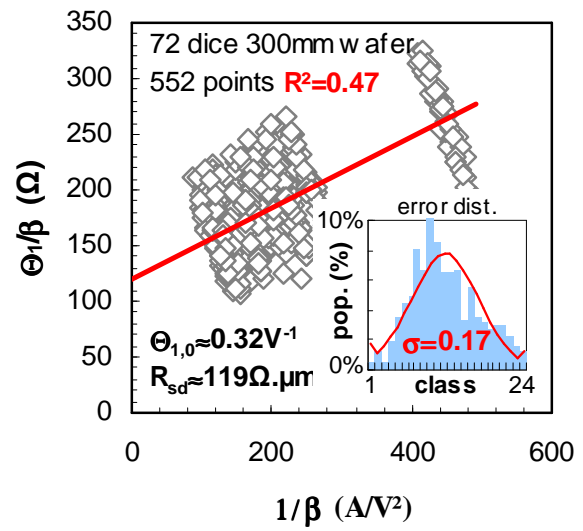

Fig.9 $-\mathrm{R}_{\mathrm{sd}}$. and $\Theta_{1,0}$ extracted from the $\Theta_{1}(\beta)$ technique. In inset: error distribution

\section{References}

[1] The ITRS, Semiconductor Ind. Assoc., 2006.

[2] K.M. Cao et al., IEDM.1999 pp. 171-174

[3] F. Andrieu et al., IEEE EDL, Oct 2005

[4] A. Cros et al., IEDM.2006 pp. 663-666

[5] Y. Taur et al., IEEE EDL, May 1992

[6] G.J. Hu et al., IEEE TED, Dec 1997

[7] Y.H. Chang et al., EDSSC 2007, pp.87-90

[8] J. Kim et al., IEEE TED, Oct 2008

[9] E. Josse et al., IEDM 2006, pp. 693

[10] D. Aimé et al., ESSDERC 2007, pp.255-258

[11] P.K. McLarty et al., SSE, Jun 1995

[12] D. Fleury et al., ICMTS 2008, pp.160-165

[13] D. Fleury et al., IEEE T-SM, Nov 2008

[14] S.D. Kim et al, IEEE TED, Mar2002

[15] G. Ghibaudo, Electronics Letters, Apr 1988

[16] C.Mourrain et al, ICMTS 2000, pp.181-186 